\title{
Reaction between thiocarbamidoalkyl naphthols and acetylenic esters: An interesting cyclocondensation reaction for the synthesis of new thiazolidin-4-one derivatives
}

\author{
SAKINEH AMINI*, AHMAD MOMENI TIKDARI and HOJATOLLAH KHABAZZADEH \\ Department of Chemistry, Shahid Bahonar University of Kerman, Kerman 7616914111, Iran \\ e-mail: h-amini@sci.uk.ac.ir
}

MS received 8 February 2015; revised 5 May 2015; accepted 20 May 2015

\begin{abstract}
This investigation was set to provide derivatives of thiazolidin-4-ones incorporated with aminoalkyl naphthols in a molecular frame work. For this purpose, a series of 1-thiocarbamidoalkyl-2-naphthols was prepared by the three component condensation of aromatic aldehydes, phenylthiourea and 2-naphthol. In the next step, these compounds underwent reaction with dialkyl acetylenedicarboxylates at ambient temperature in ethanol to afford the corresponding 4-thiazolidinones in high yields. Following the completion of the reaction, the products were solidified and isolated by filtration. The method is easy, inexpensive, chemoselective and environmentally benign and illustrates an interesting instance of click chemistry.
\end{abstract}

Keywords. Multi component reaction; cyclocondensation; 1-thiocarbamidoalkyl-2-naphthols; acetylenic esters; thiazolidin-4-ones.

\section{Introduction}

During the last two centuries, multicomponent reactions have been considered as a unique and versatile tool for the creation of diverse chemical libraries of pharmaceutical agents. ${ }^{1}$ One instance of the MCRs is the synthesis of 1-amidoalkyl-2-naphthol derivatives. These compounds are a versatile class of intermediates in the synthesis of biologically active reagents such as 1, 3-oxazines and aminoalkyl naphthols. It is, therefore, not surprising that a considerable number of protocols for the synthesis of these compounds by three-component condensation of aldehydes, $\beta$ naphthol and acetonitrile or amides/carbamates or urea in the presence of different catalysts have been reported..$^{2-5}$ However, in consequence of the lower reactivity of the thio analogues of amide and urea, only limited published information regarding the replacement of amide with thioamide or thiourea are available in the literature. ${ }^{6-9}$ The thioamido- and thiocarbamidoalkyl naphthols which were produced by these methods can be used as convenient building blocks in the synthesis of sulfur and nitrogen containing heterocyclic compounds.

Thiazolidinones are an important group of five membered heterocyclic rings. The 4-thiazolidinone scaffold has featured in a number of clinically prescribed drugs. They can be used as antibacterial, ${ }^{10}$

\footnotetext{
*For correspondence
}

anti-inflammatory, ${ }^{11,12}$ anti-cancer,${ }^{13-15}$ antihistaminic, ${ }^{16}$ anti-fungal, ${ }^{17}$ anti-HIV ${ }^{18}$ and anti-hypertensive ${ }^{19}$ agents.

Due to the biological importance of these compounds, the development of efficient protocols for their preparation has received significant attention and several improved procedures have recently been reported. One of the most convenient and effective methods that has ever been designed is the reaction between thioamides and thiosemicarbazide derivatives with dialkyl acetylenedicarboxylates which provides 2-imino-5-alkoxycarbonyl-thiazolidin-4-ones..$^{20-22}$

The current study was designed to afford derivatives of thiazolidin-4-ones incorporated with aminoalkyl naphthols in a molecular frame work.

\section{Experimental}

\subsection{Materials and Methods}

All chemicals were purchased from Merck and Fluka Chemical Companies and were used without further purification. Melting points were determined using an Electrothermal-9100 melting point apparatus and were uncorrected. IR spectra were run on a Bruker Tensor-27 FT-IR spectrometer. NMR spectra were recorded on a Bruker $400-\mathrm{MHz}$ spectrometer using $\mathrm{CDCl}_{3}$ as solvent. Carbon assignments for some of products were made based on DEPT 135 spectroscopy. Elemental analyses 
were performed on a Vario Macro CHNS Element Analyzer.

\subsection{General procedure for the preparation of thiazolidin-4-ones}

To a magnetically well stirred solution of 1thiocarbamido-2-naphthol $(1 \mathrm{mmol})$ in $\mathrm{EtOH}(5 \mathrm{~mL})$, dimethyl acetylenedicarboxylate (DMAD) or diethyl acetylenedicarboxylate (DEAD), (1.2 mmol) was added at ambient temperature. The reaction mixture was then allowed to stir for an appropriate time. After the completion of reaction (as indicated by TLC, ethyl acetate: $n$-hexane, 30:70), the solid product was filtered off and washed thoroughly with cold ethanol.

\subsection{Characterization data}

2a: IR $(\mathrm{KBr}): v_{\max }=3430,3062,2955,1731,1684$, $1644 \mathrm{~cm}^{-1} ;{ }^{1} \mathrm{H}$ NMR $\left(400 \mathrm{MHz}, \mathrm{CDCl}_{3}\right) \delta: 9.81(\mathrm{~s}$, $1 \mathrm{H}, \mathrm{OH}), 7.95(\mathrm{~d}, J=8.4 \mathrm{~Hz}, 1 \mathrm{H}), 7.75(\mathrm{~d}, J=$ $7.6 \mathrm{~Hz}, 1 \mathrm{H}), 7.69(\mathrm{~d}, J=8.8 \mathrm{~Hz}, 1 \mathrm{H}), 7.64-7.54(\mathrm{~m}$, $3 \mathrm{H}), 7.50-7.46(\mathrm{~m}, 1 \mathrm{H}), 7.43-7.40(\mathrm{~m}, 2 \mathrm{H}), 7.36-7.21$ $(\mathrm{m}, 6 \mathrm{H}), 7.09(\mathrm{~s}, 1 \mathrm{H}), 7.08(\mathrm{~d}, J=9.2 \mathrm{~Hz}, 1 \mathrm{H}), 6.58$ $(\mathrm{s}, 1 \mathrm{H}), 3.94\left(\mathrm{~s}, 3 \mathrm{H}, \mathrm{OCH}_{3}\right) \mathrm{ppm} ;{ }^{13} \mathrm{C} \mathrm{NMR+DEPT}$ $\left(100 \mathrm{MHz}, \mathrm{CDCl}_{3}\right) \delta: 166.4(\mathrm{C}), 164.0(\mathrm{C}), 154.7$ (C), 153.6 (C), 140.6 (C), 139.7 (C), 133.5 (C), 131.6 (C), $130.0(\mathrm{CH}), 129.9(\mathrm{CH}), 129.8(\mathrm{CH}), 128.9(\mathrm{CH}), 128.8$ $(\mathrm{CH}), 128.7(\mathrm{C}), 127.9(\mathrm{CH}), 127.8(\mathrm{CH}), 127.5(\mathrm{CH})$, $127.0(\mathrm{CH}), 122.9(\mathrm{CH}), 121.4(\mathrm{CH}), 120.3(\mathrm{CH}), 117.6$ $(\mathrm{CH}), 115.8(\mathrm{C}), 66.1(\mathrm{CH}), 52.9\left(\mathrm{CH}_{3}\right)$ ppm. Anal. Calc. for $\mathrm{C}_{29} \mathrm{H}_{22} \mathrm{~N}_{2} \mathrm{O}_{4} \mathrm{~S}: \mathrm{C} 70.43, \mathrm{H} 4.48, \mathrm{~N}$ 5.66, S 6.48\%. Found: C 70.83, H 4.55, N 5.41, S 6.53\%.

2b: IR (KBr): $v_{\max }=3439,3072,3007,1725,1705$, $1649 \mathrm{~cm}^{-1} ;{ }^{1} \mathrm{H}$ NMR $\left(400 \mathrm{MHz}, \mathrm{CDCl}_{3}\right) \delta: 9.15(\mathrm{~s}, 1 \mathrm{H}$, $\mathrm{OH}), 7.71(\mathrm{~d}, J=8.4 \mathrm{~Hz}, 1 \mathrm{H}), 7.65\left(\mathrm{dd}, J_{1}=11.6 \mathrm{~Hz}\right.$, $\left.J_{2}=8.8 \mathrm{~Hz}, 2 \mathrm{H}\right), 7.50-7.34(\mathrm{~m}, 5 \mathrm{H}), 7.25-7.19(\mathrm{~m}$, $3 \mathrm{H}), 7.06(\mathrm{~d}, J=8.4 \mathrm{~Hz}, 1 \mathrm{H}), 7.00(\mathrm{~d}, J=8.8 \mathrm{~Hz}$, $1 \mathrm{H}), 6.96(\mathrm{~s}, 1 \mathrm{H}), 6.93\left(\mathrm{dd}, J_{1}=8.4 \mathrm{~Hz}, J_{2}=2 \mathrm{~Hz}\right.$, 1H), $6.75(\mathrm{~s}, 1 \mathrm{H}), 3.79$ (s, 3H, OC $\left.\mathrm{H}_{3}\right) \mathrm{ppm} ;{ }^{13} \mathrm{C} \mathrm{NMR}$ $\left(100 \mathrm{MHz}, \mathrm{CDCl}_{3}\right) \delta: 166.3,164.2,155.1,154.9,139.8$, 136.1, 134.6, 133.9, 133.4, 131.4, 131.2, 130.6, 129.9, $129.8,129.6,128.8,128.7,128.0,127.9,127.5,123.3$, 121.1, 120.0, 117.5, 115.2, 62.7, 52.9 ppm. Anal. Calc. for $\mathrm{C}_{29} \mathrm{H}_{20} \mathrm{Cl}_{2} \mathrm{~N}_{2} \mathrm{O}_{4} \mathrm{~S}: \mathrm{C} 61.82, \mathrm{H} 3.58, \mathrm{~N} 4.97$, S 5.69\%. Found: C 61.99, H 3.55, N 4.69, S 6.69\%.

2c: IR (KBr): $v_{\max }=3483,3062,2949,1730,1691$, $1646 \mathrm{~cm}^{-1} ;{ }^{1} \mathrm{H}$ NMR $\left(400 \mathrm{MHz}, \mathrm{CDCl}_{3}\right) \delta: 9.72(\mathrm{~s}$, $1 \mathrm{H}, \mathrm{OH}), 7.88(\mathrm{~d}, J=8.4 \mathrm{~Hz}, 1 \mathrm{H}), 7.76(\mathrm{~d}, J=$ $7.6 \mathrm{~Hz}, 1 \mathrm{H}), 7.70(\mathrm{~d}, J=9.2 \mathrm{~Hz}, 1 \mathrm{H}), 7.64-7.54(\mathrm{~m}$, $3 \mathrm{H}), 7.51-7.46(\mathrm{~m}, 1 \mathrm{H}), 7.34-7.30(\mathrm{~m}, 5 \mathrm{H}), 7.24(\mathrm{td}$, $\left.J_{1}=8.4 \mathrm{~Hz}, J_{2}=2 \mathrm{~Hz}, 2 \mathrm{H}\right), 7.10(\mathrm{~s}, 1 \mathrm{H}), 7.07(\mathrm{~d}$,
$J=9.2 \mathrm{~Hz}, 1 \mathrm{H}), 6.54(\mathrm{~s}, 1 \mathrm{H}), 3.94\left(\mathrm{~s}, 3 \mathrm{H}, \mathrm{OCH}_{3}\right) \mathrm{ppm}$; ${ }^{13} \mathrm{C}$ NMR $\left(100 \mathrm{MHz}, \mathrm{CDCl}_{3}\right) \delta: 166.4,163.9,154.7$, 154.0, 139.4, 139.1, 133.8, 133.4, 131.4, 130.2, 129.9, 129.1, 128.9, 128.8, 128.7, 127.7, 127.1, 123.1, 121.2, 120.3, 117.8, 115.3, 65.3, 53.0 ppm. Anal. Calc. for $\mathrm{C}_{29} \mathrm{H}_{21} \mathrm{ClN}_{2} \mathrm{O}_{4} \mathrm{~S}: \mathrm{C}$ 65.84, H 4.00, N 5.30, S 6.06\%. Found: C 66.30, H 3.90, N 4.88, S 6.51\%.

2d: IR (KBr): $v_{\max }=3477,3145,3062,2949,1726$, $1709,1631 \mathrm{~cm}^{-1} ;{ }^{1} \mathrm{H}$ NMR $\left(400 \mathrm{MHz}, \mathrm{CDCl}_{3}\right) \delta: 9.35$ $(\mathrm{s}, 1 \mathrm{H}, \mathrm{OH}), 7.88(\mathrm{~d}, J=8.4 \mathrm{~Hz}, 1 \mathrm{H}), 7.77-7.72(\mathrm{~m}$, $2 \mathrm{H}), 7.59-7.42(\mathrm{~m}, 5 \mathrm{H}), 7.34-7.31(\mathrm{~m}, 3 \mathrm{H}), 7.25(\mathrm{dd}$, $\left.J_{1}=8.0 \mathrm{~Hz}, J_{2}=1.2 \mathrm{~Hz}, 1 \mathrm{H}\right), 7.18\left(\mathrm{dt}, J_{1}=8.0 \mathrm{~Hz}\right.$, $\left.J_{2}=1.6 \mathrm{~Hz}, 1 \mathrm{H}\right), 7.11(\mathrm{~d}, J=8.8 \mathrm{~Hz}, 1 \mathrm{H}), 7.07(\mathrm{~d}, J$ $=6.8 \mathrm{~Hz}, 1 \mathrm{H}), 7.05(\mathrm{~s}, 1 \mathrm{H}), 6.92(\mathrm{~s}, 1 \mathrm{H}), 3.90(\mathrm{~s}, 3 \mathrm{H}$, $\left.\mathrm{OCH}_{3}\right) \mathrm{ppm} ;{ }^{13} \mathrm{C} \mathrm{NMR}+\mathrm{DEPT}\left(100 \mathrm{MHz}, \mathrm{CDCl}_{3}\right) \delta$ : 166.3 (C), 164.2 (C), 154.9 (C), 154.7 (C), 140.0 (C), $137.4(\mathrm{C}), 133.5(\mathrm{C}), 133.2(\mathrm{C}), 131.6(\mathrm{C}), 130.3(\mathrm{CH})$, $130.2(\mathrm{CH}), 129.9(\mathrm{CH}), 129.8(\mathrm{CH}), 129.7(\mathrm{CH}), 129.4$ $(\mathrm{CH}), 128.8(\mathrm{CH}), 128.7(\mathrm{C}), 127.9(\mathrm{CH}), 127.7(\mathrm{CH})$, $127.3(\mathrm{CH}), 123.2(\mathrm{CH}), 121.4(\mathrm{CH}), 120.0(\mathrm{CH}), 117.3$ $(\mathrm{CH}), 115.7(\mathrm{C}), 63.3(\mathrm{CH}), 52.8\left(\mathrm{CH}_{3}\right) \mathrm{ppm}$. Anal. Calc. for: $\mathrm{C}_{29} \mathrm{H}_{21} \mathrm{ClN}_{2} \mathrm{O}_{4} \mathrm{~S}: \mathrm{C}$ 65.84, H 4.00, N 5.30, S $6.06 \%$. Found: C 65.75, H 4.10, N 5.32, S 6.56\%.

2e: IR (KBr): $v_{\max }=3473,3193,3071,2972,1724$, 1707, 1688, $1648 \mathrm{~cm}^{-1} ;{ }^{1} \mathrm{H}$ NMR $\left(400 \mathrm{MHz}, \mathrm{CDCl}_{3}\right) \delta$ : $9.36(\mathrm{~s}, 1 \mathrm{H}, \mathrm{OH}), 8.25(\mathrm{~d}, J=2.0 \mathrm{~Hz}, 1 \mathrm{H}), 8.09(\mathrm{dd}$, $\left.J_{1}=8.0 \mathrm{~Hz}, J_{2}=1.2 \mathrm{~Hz}, 1 \mathrm{H}\right), 7.93(\mathrm{~d}, J=8.4 \mathrm{~Hz}$, $1 \mathrm{H}), 7.79(\mathrm{~d}, J=7.6 \mathrm{~Hz}, 1 \mathrm{H}), 7.74(\mathrm{~d}, J=8.8 \mathrm{~Hz}, 1 \mathrm{H})$, 7.66-7.51 (m, 5H), $7.41(\mathrm{t}, J=8.0 \mathrm{~Hz}, 1 \mathrm{H}), 7.38-7.34$ $(\mathrm{m}, 3 \mathrm{H}), 7.11(\mathrm{~s}, 1 \mathrm{H}), 7.09(\mathrm{~d}, J=8.8 \mathrm{~Hz}, 1 \mathrm{H}), 6.67$ (s, $1 \mathrm{H}), 3.93$ (s, $\left.3 \mathrm{H}, \mathrm{OCH}_{3}\right) \mathrm{ppm} ;{ }^{13} \mathrm{C}$ NMR $(100 \mathrm{MHz}$, $\left.\mathrm{CDCl}_{3}\right) \delta: 166.3,163.9,154.7,148.4,142.8,133.4$, 131.4, 130.6, 130.0, 129.9, 129.1, 128.8, 127.7, 127.5, 123.3, 122.9, 122.1, 120.3, 118.2, 65.9, 53.0 ppm. Anal. Calc. for $\mathrm{C}_{29} \mathrm{H}_{21} \mathrm{~N}_{3} \mathrm{O}_{6} \mathrm{~S} ; \mathrm{C}$ 64.55, H 3.92, N 7.79, S 5.94\%. Found: C 64.03, H 4.21, N 7.54, S $5.66 \%$.

2f: IR (KBr): $v_{\max }=3480,3062,2953,1715,1691$, $1642 \mathrm{~cm}^{-1} ;{ }^{1} \mathrm{H} \mathrm{NMR}\left(400 \mathrm{MHz}, \mathrm{CDCl}_{3}\right) \delta: 9.51(\mathrm{~s}, 1 \mathrm{H}$, $\mathrm{OH}), 8.12\left(\mathrm{td}, J_{1}=9.2 \mathrm{~Hz}, J_{2}=2.0 \mathrm{~Hz}, 2 \mathrm{H}\right), 7.89$ $(\mathrm{d}, J=8.4 \mathrm{~Hz}, 1 \mathrm{H}), 7.78(\mathrm{~d}, J=7.2 \mathrm{~Hz}, 1 \mathrm{H}), 7.73(\mathrm{~d}$, $J=8.8 \mathrm{~Hz}, 1 \mathrm{H}), 7.65-7.49(\mathrm{~m}, 7 \mathrm{H}), 7.36-7.33(\mathrm{~m}, 3 \mathrm{H})$, $7.11(\mathrm{~s}, 1 \mathrm{H}), 7.08(\mathrm{~d}, J=8.8 \mathrm{~Hz}, 1 \mathrm{H}), 6.66(\mathrm{~s}, 1 \mathrm{H}), 3.93$ $\left(\mathrm{s}, 3 \mathrm{H}, \mathrm{OCH}_{3}\right) \mathrm{ppm} ;{ }^{13} \mathrm{C} \mathrm{NMR}\left(100 \mathrm{MHz}, \mathrm{CDCl}_{3}\right) \delta$ : $166.3,163.8,154.7,154.6,147.8,147.7,147.3,139.2$, $133.3,131.3,130.6,130.0,129.9,129.1,128.8,128.2$, 127.7, 127.4, 124.1, 123.3, 121.0, 120.3, 118.2, 64.6, 53.0 ppm. Anal. Calc. for $\mathrm{C}_{29} \mathrm{H}_{21} \mathrm{~N}_{3} \mathrm{O}_{6} \mathrm{~S} ; \mathrm{C} 64.55, \mathrm{H}$ 3.92, N 7.79, S 5.94\%. Found: C 64.55, H 4.40, N 7.39, S $5.55 \%$.

2g: IR (KBr): $v_{\max }=3386,3061,2949,1730,1690$, $1643 \mathrm{~cm}^{-1} ;{ }^{1} \mathrm{H}$ NMR $\left(400 \mathrm{MHz}, \mathrm{CDCl}_{3}\right) \delta: 9.87(\mathrm{~s}, 1 \mathrm{H}$, 
$\mathrm{OH}), 7.91(\mathrm{~d}, J=8.4 \mathrm{~Hz}, 1 \mathrm{H}), 7.74(\mathrm{~d}, J=7.6 \mathrm{~Hz}$, $1 \mathrm{H}), 7.68(\mathrm{~d}, J=8.8 \mathrm{~Hz}, 1 \mathrm{H}), 7.63-7.53(\mathrm{~m}, 3 \mathrm{H}), 7.49-$ $7.45(\mathrm{~m}, 1 \mathrm{H}), 7.35-7.30(\mathrm{~m}, 5 \mathrm{H}), 7.09(\mathrm{~s}, 1 \mathrm{H}), 7.07(\mathrm{~d}$, $J=8.8 \mathrm{~Hz}, 1 \mathrm{H}), 6.80\left(\mathrm{td}, J_{1}=8.8 \mathrm{~Hz}, J_{2}=2 \mathrm{~Hz}\right.$, $2 \mathrm{H}), 6.53(\mathrm{~s}, 1 \mathrm{H}), 3.94\left(\mathrm{~s}, 3 \mathrm{H}, \mathrm{OCH}_{3}\right), 3.74(\mathrm{~s}, 3 \mathrm{H}$, $\left.\mathrm{OCH}_{3}\right) \mathrm{ppm} ;{ }^{13} \mathrm{C} \mathrm{NMR}+\mathrm{DEPT}\left(100 \mathrm{MHz}, \mathrm{CDCl}_{3}\right) \delta$ : 166.4 (C), 163.9 (C), 159.1 (C), 154.6 (C), 153.2 (C), 139.7 (C), 133.5 (C), 132.8 (C), $131.5(\mathrm{C}), 129.9(\mathrm{CH})$, $129.8(\mathrm{CH}), 128.9,128.8(\mathrm{CH}), 128.7(\mathrm{CH}), 127.8$ $(\mathrm{CH}), 127.0(\mathrm{CH}), 122.9(\mathrm{CH}), 121.4(\mathrm{CH}), 120.3(\mathrm{CH})$, $117.5(\mathrm{CH}), 115.9(\mathrm{C}), 114.2(\mathrm{CH}), 65.7(\mathrm{CH}), 55.2$ $\left(\mathrm{CH}_{3}\right), 52.9\left(\mathrm{CH}_{3}\right)$ ppm. Anal. Calc. for $\mathrm{C}_{30} \mathrm{H}_{24} \mathrm{~N}_{2} \mathrm{O}_{5} \mathrm{~S}$ : C 68.69, H 4.61, N 5.34, S 6.11\%. Found: C 68.36, H 4.99 , N 5.70, S $6.27 \%$.

2h: IR (KBr): $v_{\max }=3374,3061,2949,1731,1690$, $1644 \mathrm{~cm}^{-1} ;{ }^{1} \mathrm{H} \mathrm{NMR}\left(400 \mathrm{MHz}, \mathrm{CDCl}_{3}\right) \delta: 10.0(\mathrm{~s}, 1 \mathrm{H}$, $\mathrm{OH}), 7.90(\mathrm{~d}, J=8.4 \mathrm{~Hz}, 1 \mathrm{H}), 7.74(\mathrm{~d}, J=7.2 \mathrm{~Hz}, 1 \mathrm{H})$, $7.68(\mathrm{~d}, J=8.8 \mathrm{~Hz}, 1 \mathrm{H}), 7.57-7.44(\mathrm{~m}, 4 \mathrm{H}), 7.32-7.29$ $(\mathrm{m}, 3 \mathrm{H}), 7.25\left(\mathrm{td}, J_{1}=8.8 \mathrm{~Hz}, J_{2}=2.8 \mathrm{~Hz}, 2 \mathrm{H}\right), 7.09$ $(\mathrm{s}, 1 \mathrm{H}), 7.06(\mathrm{~d}, J=8.8 \mathrm{~Hz}, 1 \mathrm{H}), 6.69\left(\mathrm{td}, J_{1}=8.8 \mathrm{~Hz}\right.$, $\left.J_{2}=2.8 \mathrm{~Hz}, 2 \mathrm{H}\right), 6.51(\mathrm{~s}, 1 \mathrm{H}), 5.68(\mathrm{~s}, 1 \mathrm{H}, \mathrm{OH}), 3.94$ (s, $\left.3 \mathrm{H}, \mathrm{OCH}_{3}\right) \mathrm{ppm} ;{ }^{13} \mathrm{C} \mathrm{NMR}\left(100 \mathrm{MHz}, \mathrm{CDCl}_{3}\right) \delta$ : 166.5, 164.0, 155.5, 154.4, 153.3, 139.8, 133.4, 132.6, $131.5,129.9,128.9,128.8,128.7,127.7,127.0,123.0$, 121.5, 120.2, 117.6, 115.9, 115.7, 65.7, 53.0 ppm. Anal. Calc. for $\mathrm{C}_{29} \mathrm{H}_{22} \mathrm{~N}_{2} \mathrm{O}_{5} \mathrm{~S}: \mathrm{C}$ 68.22, H 4.34, N 5.49, S 6.28\%. Found: C 68.11, H 4.18, N 5.47, S 5.56\%.

2i: IR (KBr): $v_{\max }=3456,3141,3071,1726,1680$, $1639 \mathrm{~cm}^{-1} ;{ }^{1} \mathrm{H}$ NMR $\left(400 \mathrm{MHz}, \mathrm{CDCl}_{3}\right) \delta: 9.84(\mathrm{~s}, 1 \mathrm{H}$, $\mathrm{OH}), 7.94(\mathrm{~d}, J=8.8 \mathrm{~Hz}, 1 \mathrm{H}), 7.75(\mathrm{~d}, J=7.2 \mathrm{~Hz}$, $1 \mathrm{H}), 7.69(\mathrm{~d}, J=9.2 \mathrm{~Hz}, 1 \mathrm{H}), 7.63-7.54(\mathrm{~m}, 3 \mathrm{H})$, 7.50-7.40 (m, 3H), 7.35-7.30 (m, 4H), 7.27-7.21 (m, 2H), 7.09 (s, 1H), 7.07 (s, 1H), $6.58(\mathrm{~s}, 1 \mathrm{H}), 4.39$ (q, $\left.J=7.2 \mathrm{~Hz}, 2 \mathrm{H}, \mathrm{OCH}_{2} \mathrm{CH}_{3}\right), 1.41(\mathrm{t}, J=7.2 \mathrm{~Hz}, 3 \mathrm{H}$, $\left.\mathrm{OCH}_{2} \mathbf{C} H_{3}\right)$ ppm; ${ }^{13} \mathrm{C} \mathrm{NMR+DEPT}\left(100 \mathrm{MHz}, \mathrm{CDCl}_{3}\right)$ $\delta: 166.0(\mathrm{C}), 164.0(\mathrm{C}), 154.7(\mathrm{C}), 153.8$ (C), 140.6 (C), $139.4(\mathrm{C}), 133.5(\mathrm{C}), 131.6(\mathrm{C}), 130.0(\mathrm{CH}), 129.9$ $(\mathrm{CH}), 129.8(\mathrm{CH}), 128.9(\mathrm{CH}), 128.8(\mathrm{CH}), 128.7(\mathrm{C})$, 127.9 $(\mathrm{CH}), 127.8(\mathrm{CH}), 127.5(\mathrm{CH}), 127.0(\mathrm{CH}), 122.9$ $(\mathrm{CH}), 121.4(\mathrm{CH}), 120.3(\mathrm{CH}), 118.1(\mathrm{CH}), 115.8(\mathrm{C})$, $66.1(\mathrm{CH}), 62.2\left(\mathrm{CH}_{2}\right), 14.2\left(\mathrm{CH}_{3}\right)$ ppm; Anal. Calc. for $\mathrm{C}_{30} \mathrm{H}_{24} \mathrm{~N}_{2} \mathrm{O}_{4} \mathrm{~S}$ : C 70.85, H 4.76, N 5.51, S 6.30\%. Found: C 70.37, H 4.87, N 5.42, S 6.58\%

2j: IR (KBr): $v_{\max }=3437,3070,2993,1727,1698$, $1651 \mathrm{~cm}^{-1} ;{ }^{1} \mathrm{H}$ NMR $\left(400 \mathrm{MHz}, \mathrm{CDCl}_{3}\right) \delta: 9.28(\mathrm{~s}, 1 \mathrm{H}$, $\mathrm{OH}), 7.82(\mathrm{~d}, J=8.8 \mathrm{~Hz}, 1 \mathrm{H}), 7.78-7.73(\mathrm{~m}, 2 \mathrm{H})$, 7.60-7.44 (m, 5H), 7.35-7.30 (m, 3H), $7.16(\mathrm{~d}, J=$ $8.4 \mathrm{~Hz}, 1 \mathrm{H}), 7.11(\mathrm{~d}, J=8.8 \mathrm{~Hz}, 1 \mathrm{H}), 7.05(\mathrm{~s}, 1 \mathrm{H})$, $7.03\left(\mathrm{dd}, J_{1}=8.8 \mathrm{~Hz}, J_{2}=2.0 \mathrm{~Hz}, 1 \mathrm{H}\right), 6.86(\mathrm{~s}$, $1 \mathrm{H}), 4.34\left(\mathrm{q}, J=7.2 \mathrm{~Hz}, 2 \mathrm{H}, \mathrm{OCH}_{2} \mathrm{CH}_{3}\right), 1.37(\mathrm{t}, J=$ $\left.7.2 \mathrm{~Hz}, 3 \mathrm{H}, \mathrm{OCH}_{2} \mathrm{CH}_{3}\right)$ ppm.; ${ }^{13} \mathrm{C} \mathrm{NMR}(100 \mathrm{MHz}$,
$\left.\mathrm{CDCl}_{3}\right) \delta: 165.8,164.2,155.3,154.9,139.4,136.1$, 134.6, 133.9, 133.5, 131.4, 131.2, 130.6, 129.9, 129.8, $129.6,128.8,128.7,128.0,127.9,127.5,123.3,121.1$, 120.0, 118.1, 115.3, 62.7, 62.1, 14.2 ppm; Anal. Calc. for $\mathrm{C}_{30} \mathrm{H}_{22} \mathrm{Cl}_{2} \mathrm{~N}_{2} \mathrm{O}_{4} \mathrm{~S}$; C 62.40, H 3.84, N 4.85, S 5.55. Found: C 62.74, H 3.77, N 4.48, S 5.35\%.

2k: IR (KBr): $v_{\max }=3434,3068,2985,1726,1699$, $1652 \mathrm{~cm}^{-1} ;{ }^{1} \mathrm{H}$ NMR $\left(400 \mathrm{MHz}, \mathrm{CDCl}_{3}\right) \delta: 9.74(\mathrm{~s}, 1 \mathrm{H}$, $\mathrm{OH}), 7.88(\mathrm{~d}, J=8.4 \mathrm{~Hz}, 1 \mathrm{H}), 7.76(\mathrm{~d}, J=8.0 \mathrm{~Hz}, 1 \mathrm{H})$, $7.70(\mathrm{~d}, J=8.8 \mathrm{~Hz}, 1 \mathrm{H}), 7.64-7.54(\mathrm{~m}, 3 \mathrm{H}), 7.50-7.46$ (m, 1H), 7.35-7.30 (m, 5H), $7.25\left(\mathrm{td}, J_{1}=8.4 \mathrm{~Hz}, J_{2}=\right.$ $2.4 \mathrm{~Hz}, 2 \mathrm{H}), 7.10(\mathrm{~s}, 1 \mathrm{H}), 7.07(\mathrm{~d}, J=8.8 \mathrm{~Hz}, 1 \mathrm{H}), 6.55$ (s, $1 \mathrm{H}), 4.39$ (q, $\left.J=7.2 \mathrm{~Hz}, 2 \mathrm{H}, \mathrm{OCH}_{2} \mathrm{CH}_{3}\right), 1.41$ (t, $\left.J=7.2 \mathrm{~Hz}, 3 \mathrm{H}, \mathrm{OCH}_{2} \mathrm{CH}_{3}\right) \mathrm{ppm} ;{ }^{13} \mathrm{C} \mathrm{NMR}+\mathrm{DEPT}$ $\left(100 \mathrm{MHz}, \mathrm{CDCl}_{3}\right) \delta: 166.0$ (C), 164.0 (C), 154.7 (C), 154.2 (C), 139.2 (C), 139.1 (C), 133.7 (C), 133.4 (C), $131.4(\mathrm{C}), 130.2(\mathrm{CH}), 129.9(\mathrm{CH}), 129.1(\mathrm{CH}), 128.9$ $(\mathrm{CH}), 128.8(\mathrm{CH}), 128.7(\mathrm{C}), 127.7(\mathrm{CH}), 127.1(\mathrm{CH})$, $123.1(\mathrm{CH}), 121.2(\mathrm{CH}), 120.3(\mathrm{CH}), 118.3(\mathrm{CH}), 115.4$ (C), $65.3(\mathrm{CH}), 62.2\left(\mathrm{CH}_{2}\right), 14.2\left(\mathrm{CH}_{3}\right) \mathrm{ppm}$; Anal. Calc. for $\mathrm{C}_{30} \mathrm{H}_{23} \mathrm{ClN}_{2} \mathrm{O}_{4} \mathrm{~S} ; \mathrm{C} 66.35, \mathrm{H} 4.27, \mathrm{~N} 5.16, \mathrm{~S}$ 5.90\%. Found: C 66.55, H 4.15, N 5.03, S 5.61\%.

2I: IR (KBr): $v_{\max }=3430,3065,2983,1726,1697$, $1637 \mathrm{~cm}^{-1} ;{ }^{1} \mathrm{H}$ NMR $\left(400 \mathrm{MHz}, \mathrm{CDCl}_{3}\right) \delta: 9.36(\mathrm{~s}, 1 \mathrm{H}$, $\mathrm{OH}), 7.88(\mathrm{~d}, J=8.4 \mathrm{~Hz}, 1 \mathrm{H}), 7.76(\mathrm{~d}, J=8.4 \mathrm{~Hz}$, $1 \mathrm{H}), 7.73(\mathrm{~d}, J=8.8 \mathrm{~Hz}, 1 \mathrm{H}), 7.59-7.46(\mathrm{~m}, 4 \mathrm{H}), 7.42$ $\left(\mathrm{dd}, J_{1}=8.0 \mathrm{~Hz}, J_{2}=0.8 \mathrm{~Hz}, 1 \mathrm{H}\right), 7.34-7.30(\mathrm{~m}, 3 \mathrm{H})$, $7.25\left(\mathrm{dd}, J_{1}=7.6 \mathrm{~Hz}, J_{2}=1.6 \mathrm{~Hz}, 1 \mathrm{H}\right), 7.18(\mathrm{dt}$, $\left.J_{1}=8.0 \mathrm{~Hz}, J_{2}=1.6 \mathrm{~Hz}, 1 \mathrm{H}\right), 7.11(\mathrm{~d}, J=8.8 \mathrm{~Hz}$, $1 \mathrm{H}), 7.07\left(\mathrm{dd}, J_{1}=7.6 \mathrm{~Hz}, J_{2}=1.2 \mathrm{~Hz}, 1 \mathrm{H}\right), 7.04(\mathrm{~s}$, $1 \mathrm{H}), 6.91$ (s, 1H), 4.34 (q, $J=7.2 \mathrm{~Hz}, 2 \mathrm{H}, \mathrm{OCH}_{2} \mathrm{CH}_{3}$ ), $1.37\left(\mathrm{t}, J=7.2 \mathrm{~Hz}, 3 \mathrm{H}, \mathrm{OCH}_{2} \mathrm{CH}_{3}\right) \mathrm{ppm} ;{ }^{13} \mathrm{C} \mathrm{NMR}$ $\left(100 \mathrm{MHz}, \mathrm{CDCl}_{3}\right) \delta: 165.8,164.3,154.9,154.8,139.7$, $137.5,133.5,133.2,131.6,130.3,130.2,129.9,129.8$, $129.7,129.4,128.8,128.7,127.9,127.7,127.3,123.2$, 121.4, 120.0, 117.9, 115.7, 63.3, 62.0, 14.2 ppm; Anal. Calc. for $\mathrm{C}_{30} \mathrm{H}_{23} \mathrm{ClN}_{2} \mathrm{O}_{4} \mathrm{~S}$ : C 66.35, H 4.27, N 5.16, S 5.90\%. Found: C 66.88, H 4.27, N 5.03, S 5.75\%.

2m: IR (KBr): $v_{\max }=3516,3078,2978,1732,1697$, $1652 \mathrm{~cm}^{-1} ;{ }^{1} \mathrm{H}$ NMR $\left(400 \mathrm{MHz}, \mathrm{CDCl}_{3}\right) \delta: 9.39(\mathrm{~s}$, $1 \mathrm{H}, \mathrm{OH}), 8.25(\mathrm{t}, J=2.0 \mathrm{~Hz}, 1 \mathrm{H}), 8.11-8.08(\mathrm{~m}, 1 \mathrm{H})$, $7.93(\mathrm{~d}, J=8.4 \mathrm{~Hz}, 1 \mathrm{H}), 7.79(\mathrm{~d}, J=7.6 \mathrm{~Hz}, 1 \mathrm{H})$, $7.74(\mathrm{~d}, J=8.8 \mathrm{~Hz}, 1 \mathrm{H}), 7.66-7.51(\mathrm{~m}, 5 \mathrm{H}), 7.41$ $(\mathrm{t}, J=8.0 \mathrm{~Hz}, 1 \mathrm{H}) 7.38-7.34(\mathrm{~m}, 3 \mathrm{H}), 7.11(\mathrm{~s}, 1 \mathrm{H})$, $7.09(\mathrm{~d}, J=8.8 \mathrm{~Hz}, 1 \mathrm{H}), 6.67(\mathrm{~s}, 1 \mathrm{H}), 4.39(\mathrm{q}, J=$ $\left.7.2 \mathrm{~Hz}, 2 \mathrm{H}, \mathrm{OCH}_{2} \mathrm{CH}_{3}\right), 1.40(\mathrm{t}, J=7.2 \mathrm{~Hz}, 3 \mathrm{H}$, $\left.\mathrm{OCH}_{2} \mathrm{CH}_{3}\right) \mathrm{ppm} ;{ }^{13} \mathrm{C} \mathrm{NMR}+\mathrm{DEPT}\left(100 \mathrm{MHz}, \mathrm{CDCl}_{3}\right)$ $\delta$ : $165.8(\mathrm{C}), 164.0(\mathrm{C}), 155.3(\mathrm{C}), 154.8$ (C), 154.7 (C), $148.4(\mathrm{C}), 142.8(\mathrm{C}), 138.8(\mathrm{C}), 133.4(\mathrm{CH}), 131.4$ (C), $130.6(\mathrm{CH}), 130.0(\mathrm{CH}), 129.9(\mathrm{CH}), 129.1(\mathrm{CH})$, $128.8(\mathrm{C}), 127.7(\mathrm{CH}), 127.5(\mathrm{CH}), 123.3(\mathrm{CH}), 122.9$ 
$(\mathrm{CH}), 122.1(\mathrm{CH}), 120.9(\mathrm{CH}), 120.3(\mathrm{CH}), 118.8$ $(\mathrm{CH}), 64.2(\mathrm{CH}), 62.3\left(\mathrm{CH}_{2}\right), 14.2\left(\mathrm{CH}_{3}\right)$ ppm; Anal. Calc. for $\mathrm{C}_{30} \mathrm{H}_{23} \mathrm{~N}_{3} \mathrm{O}_{6} \mathrm{~S} ; \mathrm{C}$ 65.09, H 4.19, N 7.59, S 5.79\%.Found: C 65.16, H 4.38, N 7.52, S 5.43\%.

2n: IR (KBr): $v_{\max }=3434,3069,2981,1728,1698$, $1649 \mathrm{~cm}^{-1} ;{ }^{1} \mathrm{H}$ NMR $\left(400 \mathrm{MHz}, \mathrm{CDCl}_{3}\right) \delta: 9.54(\mathrm{~s}, 1 \mathrm{H}$, $\mathrm{OH}), 8.14-8.10(\mathrm{~m}, 2 \mathrm{H}), 7.90(\mathrm{~d}, J=8.4 \mathrm{~Hz}, 1 \mathrm{H}), 7.78$ $(\mathrm{d}, J=7.6 \mathrm{~Hz}, 1 \mathrm{H}), 7.73(\mathrm{~d}, J=8.8 \mathrm{~Hz}, 1 \mathrm{H}), 7.65-$ $7.50(\mathrm{~m}, 6 \mathrm{H}), 7.37-7.32(\mathrm{~m}, 3 \mathrm{H}), 7.11(\mathrm{~s}, 1 \mathrm{H}), 7.08$ $(\mathrm{d}, J=8.8 \mathrm{~Hz}, 1 \mathrm{H}), 6.66(\mathrm{~s}, 1 \mathrm{H}), 4.39(\mathrm{q}, J=7.2 \mathrm{~Hz}$, $\left.2 \mathrm{H}, \mathrm{OCH}_{2} \mathrm{CH}_{3}\right), 1.40\left(\mathrm{t}, J=7.2 \mathrm{~Hz}, 3 \mathrm{H}, \mathrm{OCH}_{2} \mathrm{CH}_{3}\right)$ ppm; ${ }^{13} \mathrm{C} \mathrm{NMR+DEPT}\left(100 \mathrm{MHz}, \mathrm{CDCl}_{3}\right) \delta: 165.9$ (C), 163.9 (C), 155.3 (C), 154.7 (C), 147.7 (C), 147.4 (C), $138.8(\mathrm{C}), 133.3(\mathrm{C}), 131.3(\mathrm{C}), 130.6(\mathrm{CH})$, $130.1(\mathrm{CH}), 130.0(\mathrm{CH}), 129.1(\mathrm{CH}), 128.8,128.2$ $(\mathrm{CH}), 127.7(\mathrm{CH}), 127.4(\mathrm{CH}), 124.1(\mathrm{CH}), 123.3$ $(\mathrm{CH}), 120.9(\mathrm{CH}), 120.3(\mathrm{CH}), 118.7(\mathrm{CH}), 115.0(\mathrm{C})$, $64.6(\mathrm{CH}), 62.3\left(\mathrm{CH}_{2}\right), 14.2\left(\mathrm{CH}_{3}\right)$ ppm; Anal. Calc. for $\mathrm{C}_{30} \mathrm{H}_{23} \mathrm{~N}_{3} \mathrm{O}_{6} \mathrm{~S}: \mathrm{C}$ 65.09, H 4.19, N 7.59, S 5.79\%. Found: C 65.25, H 4.28, N 7.59, S 5.74\%.

2o: IR (KBr): $v_{\max }=3437,3066,2998,1729,1691$, $1646 \mathrm{~cm}^{-1} ;{ }^{1} \mathrm{H}$ NMR $\left(400 \mathrm{MHz}, \mathrm{CDCl}_{3}\right) \delta: 9.89(\mathrm{~s}, 1 \mathrm{H}$, $\mathrm{OH}), 7.91(\mathrm{~d}, J=8.8 \mathrm{~Hz}, 1 \mathrm{H}), 7.74(\mathrm{~d}, J=7.6 \mathrm{~Hz}$, $1 \mathrm{H}), 7.68(\mathrm{~d}, J=8.8 \mathrm{~Hz}, 1 \mathrm{H}), 7.63-7.54(\mathrm{~m}, 3 \mathrm{H}), 7.49-$ $7.44(\mathrm{~m}, 1 \mathrm{H}), 7.35-7.30(\mathrm{~m}, 5 \mathrm{H}), 7.09$ (s, 1H), 7.07 (d, $J$ $=8.8 \mathrm{~Hz}, 1 \mathrm{H}), 6.80\left(\mathrm{dt}, J_{1}=8.8 \mathrm{~Hz}, J_{2}=2.8 \mathrm{~Hz}, 2 \mathrm{H}\right)$, $6.54(\mathrm{~s}, 1 \mathrm{H}), 4.40\left(\mathrm{q}, J=7.2 \mathrm{~Hz}, 2 \mathrm{H}, \mathrm{OCH}_{2} \mathrm{CH}_{3}\right), 3.75$ (s, $\left.3 \mathrm{H}, \mathrm{OCH}_{3}\right), 1.41$ (t, $J=7.2 \mathrm{~Hz}, 3 \mathrm{H}, \mathrm{OCH}_{2} \mathrm{CH}_{3}$ ) ppm; ${ }^{13} \mathrm{C}$ NMR $\left(100 \mathrm{MHz}, \mathrm{CDCl}_{3}\right) \delta: 166.0,164.0$, 159.1, 154.6, 153.4, 139.4, 133.5, 132.8, 131.5, 129.9, $129.8,129.7,128.9,128.8,128.7,127.8,126.9,122.9$, $121.4,120.3,118.0,115.9,114.2,65.7,62.1,55.2$, 14.2 ppm; Anal. Calc. for $\mathrm{C}_{31} \mathrm{H}_{26} \mathrm{~N}_{2} \mathrm{O}_{5} \mathrm{~S}$ : C 69.13, H 4.87, N 5.20, S 5.95\%. Found: C 69.62, H 4.95, N 5.22, S $6.35 \%$.

2p: IR (KBr): $v_{\max }=3418,3067,2983,1733,1689$, $1642 \mathrm{~cm}^{-1} ;{ }^{1} \mathrm{H} \mathrm{NMR}\left(400 \mathrm{MHz}, \mathrm{CDCl}_{3}\right) \delta: 10.07(\mathrm{~s}$, $1 \mathrm{H}, \mathrm{OH}), 7.89(\mathrm{~d}, J=8.4 \mathrm{~Hz}, 1 \mathrm{H}), 7.74(\mathrm{~d}, J=7.6 \mathrm{~Hz}$, $1 \mathrm{H}), 7.68(\mathrm{~d}, J=8.8 \mathrm{~Hz}, 1 \mathrm{H}), 7.56-7.43(\mathrm{~m}, 4 \mathrm{H}), 7.32-$ $7.29(\mathrm{~m}, 3 \mathrm{H}), 7.24\left(\mathrm{td}, J_{1}=8.4 \mathrm{~Hz}, J_{2}=2.8 \mathrm{~Hz}, 2 \mathrm{H}\right)$, $7.08(\mathrm{~s}, 1 \mathrm{H}), 7.06(\mathrm{~d}, J=8.8 \mathrm{~Hz}, 1 \mathrm{H}), 6.67\left(\mathrm{td}, J_{1}=\right.$ $\left.8.4 \mathrm{~Hz}, J_{2}=2.8 \mathrm{~Hz}, 2 \mathrm{H}\right), 6.51(\mathrm{~s}, 1 \mathrm{H}), 5.54(\mathrm{~s}, 1 \mathrm{H}$, $\mathrm{OH}), 4.39\left(\mathrm{q}, J=7.2 \mathrm{~Hz}, 2 \mathrm{H}, \mathrm{OCH}_{2} \mathrm{CH}_{3}\right), 1.41(\mathrm{t}$, $\left.J=7.2 \mathrm{~Hz}, 3 \mathrm{H}, \mathrm{OCH}_{2} \mathrm{CH}_{3}\right) \mathrm{ppm} ;{ }^{13} \mathrm{C} \mathrm{NMR}+\mathrm{DEPT}$ $\left(100 \mathrm{MHz}, \mathrm{CDCl}_{3}\right) \delta: 166.1$ (C), 164.0 (C), 155.5 (C), 154.3 (C), 153.5 (C), 139.5 (C), 133.4 (C), 132.6 (C), $131.5(\mathrm{C}), 129.9(\mathrm{CH}), 128.9(\mathrm{CH}), 128.8(\mathrm{CH}), 128.7$ $(\mathrm{C}), 127.7(\mathrm{CH}), 127.0(\mathrm{CH}), 123.0(\mathrm{CH}), 121.5(\mathrm{CH})$, $120.2(\mathrm{CH}), 118.1(\mathrm{CH}), 116.0(\mathrm{C}), 115.7(\mathrm{CH}), 65.7$ $(\mathrm{CH}), 62.3\left(\mathrm{CH}_{2}\right), 14.2\left(\mathrm{CH}_{3}\right) \mathrm{ppm}$; Anal. Calc. for $\mathrm{C}_{30} \mathrm{H}_{24} \mathrm{~N}_{2} \mathrm{O}_{5} \mathrm{~S}$; C 68.69, H 4.61, N 5.34, S 6.11\%. Found: C 68.81, H 4.66, N 5.48, S 6.41\%.

\section{Results and Discussion}

In this research work, the use of 1-thiocarbamidoalkyl2-naphthol derivatives $\mathbf{1}$ as precursors in the synthesis of new 4-thiazolidinones is described. The eight required substrates (1a-1h) were synthesized according to a previously reported method $^{8}$ via three component reaction of 2-naphthol, phenylthiourea and aromatic aldehydes, containing electron-donating and electronwithdrawing groups at various positions of aromatic ring, in the presence of $p$-TSA $(10 \mathrm{~mol} \%)$ as catalyst at ambient temperature in dichloromethane (scheme 1).

After synthesizing a series of 1-thiocarbamidoalkyl2-naphthols, we turned our attention on the synthesis of thiazolidin-4-one derivatives via reaction of thiocarbamidoalkyl naphthols with acetylenic esters. In an initial study, the reaction of 1-((2-hydroxynaphthalen1-yl) (phenyl) methyl)-3-phenylthiourea (1a) with DMAD was examined in different solvents. As table 1 shows, using of ethanol as solvent gave best results, so ethanol was chosen as the solvent for this reaction.

In the first experiment, the reaction of 1-((2-hydroxynaphthalen-1-yl) (phenyl) methyl)-3-phenylthiourea (1a, $1 \mathrm{mmol}$ ) with dimethyl acetylenedicarboxylate $(1.2 \mathrm{mmol})$ as representative, was performed at room temperature with reaction time $1 \mathrm{~h}$. After completion of the reaction, the mixture was filtered and the precipitate washed thoroughly with cold ethanol. The pure solid product was isolated without need to further purification, because the excess of unreacted DMAD is soluble in $\mathrm{EtOH}$ and could easily be removed from the reaction mixture. In addition, the reaction profile is very

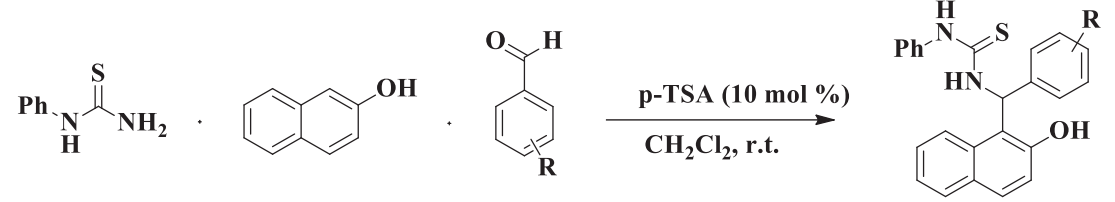

$\mathrm{R}=1 \mathrm{a}: \mathrm{H}, 1 \mathrm{~b}: 2,4(\mathrm{Cl})_{2}$, 1c: 4-Cl, 1d: 2- Cl, 1e: 3-NO , 1f: 4- $\mathrm{NO}_{2}$, 1g: 4- $\mathrm{OCH}_{3}$, 1h: 4-OH

Scheme 1. Preparation of 1-thiocarbamidoalkyl-2-naphthols. 
Table 1. Effect of solvents on product formation.

\begin{tabular}{lccc}
\hline Entry & Solvent & Time (min) & Yield (\%) \\
\hline 1 & $\mathrm{CH}_{2} \mathrm{Cl}_{2}$ & 180 & 0 \\
2 & $\mathrm{CHCl}_{3}$ & 180 & 0 \\
3 & $\mathrm{THF}$ & 180 & 80 \\
4 & EtOH & 60 & 90 \\
5 & $1,4-\mathrm{Dioxane}$ & 180 & 60 \\
6 & $\mathrm{CH}_{3} \mathrm{CN}$ & 120 & 75 \\
\hline
\end{tabular}

Table 2. Synthesis of thiazolidin-4- ones from thiocarbamidoalkyl naphthols.

\begin{tabular}{lccccc}
\hline Product & $\mathrm{R}_{1}$ & $\mathrm{R}_{2}$ & Yield (\%) & Time (min) & M.p. $\left({ }^{\circ} \mathrm{C}\right)$ \\
\hline $\mathbf{2 a}$ & $\mathrm{H}$ & $\mathrm{CH}_{3}$ & 90 & 60 & $243-245$ \\
$\mathbf{2 b}$ & 2,4-dichloro & $\mathrm{CH}_{3}$ & 85 & 60 & $212-213$ \\
$\mathbf{2 c}$ & 4-Chloro & $\mathrm{CH}_{3}$ & 91 & 90 & $187-189$ \\
$\mathbf{2 d}$ & 2-Chloro & $\mathrm{CH}_{3}$ & 92 & 75 & $146-148$ \\
$\mathbf{2}$ & $3-\mathrm{NO}_{2}$ & $\mathrm{CH}_{3}$ & 86 & 120 & $132-133$ \\
$\mathbf{2 f}$ & $4-\mathrm{NO}_{2}$ & $\mathrm{CH}_{3}$ & 93 & 120 & $143-145$ \\
$\mathbf{2 g}$ & $4-\mathrm{OCH}$ & $\mathrm{CH}_{3}$ & 89 & 75 & $203-204$ \\
$\mathbf{2 h}$ & $4-\mathrm{OH}$ & $\mathrm{CH}_{3}$ & 90 & 60 & $224-226$ \\
$\mathbf{2 i}$ & $\mathrm{H}$ & $\mathrm{CH}_{2} \mathrm{CH}_{3}$ & 81 & 180 & $171-172$ \\
$\mathbf{2 j}$ & $2,4-$ dichloro & $\mathrm{CH}_{2} \mathrm{CH}_{3}$ & 82 & 170 & $210-211$ \\
$\mathbf{2 k}$ & $4-\mathrm{Chloro}$ & $\mathrm{CH}_{2} \mathrm{CH}_{3}$ & 86 & 180 & $189-190$ \\
$\mathbf{2}$ & $2-\mathrm{Chloro}$ & $\mathrm{CH}_{2} \mathrm{CH}_{3}$ & 84 & 165 & $121-122$ \\
$\mathbf{2 m}$ & $3-\mathrm{NO}_{2}$ & $\mathrm{CH}_{2} \mathrm{CH}_{3}$ & 80 & 180 & $201-202$ \\
$\mathbf{2 n}$ & $4-\mathrm{NO}_{2}$ & $\mathrm{CH}_{2} \mathrm{CH}_{3}$ & 85 & 180 & $229-231$ \\
$\mathbf{2 0}$ & $4-\mathrm{OCH}$ & $\mathrm{CH}_{2} \mathrm{CH}_{3}$ & 86 & 165 & $203-205$ \\
$\mathbf{2 p}$ & $4-\mathrm{OH}$ & $\mathrm{CH}_{2} \mathrm{CH}_{3}$ & 81 & 150 & $223-225$ \\
\hline
\end{tabular}

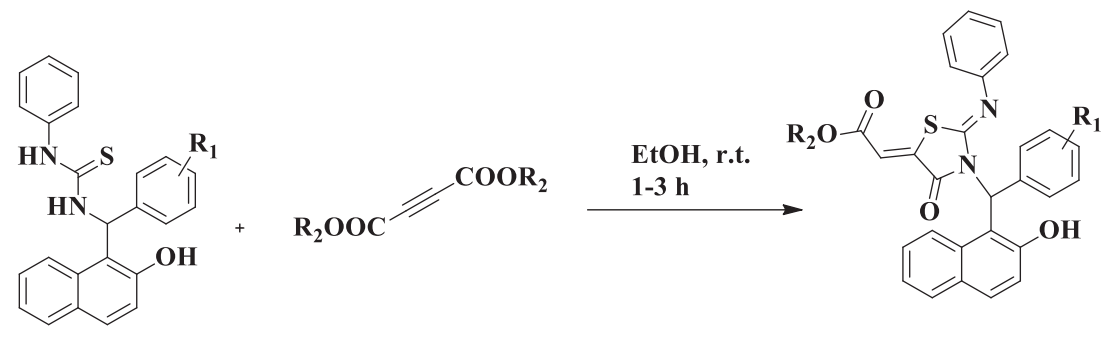

Scheme 2. Reaction of thiocarbamidoalkyl naphthols with DMAD and DEAD.

clean and no by product is formed. Encouraged by these excellent results, we examined the scope of this method for reactions of the other synthesized thiocarbamidoalkyl naphthols with DMAD. In every case, the reaction was accomplished in a short time and the corresponding product was obtained in high yield. It is pertinent to note, since some of the compounds such as $\mathbf{1 e}$ and $\mathbf{1 f}$ are less soluble in ethanol, longer reaction time (about $2 \mathrm{~h}$ ) was needed for the reaction to be completed. The difference in the product yields is mainly due to the different solubility of thiocarbamidoalkyl naphthols in ethanol which is a result of different substituents on the phenyl ring.

In a similar fashion, the reactions of thiocarbamidoalkyl naphthols with diethyl acetylenedicarboxylate have been investigated. It was found that these reactions gave lower yields of products and needed longer times to completion (2-3h) due to the low reactivity of DEAD compared to DMAD. The results are summarized in table 2 (scheme 2 ).

A plausible mechanism for the synthesis of thiazolidin-4-one derivatives has been proposed in scheme 3 . This reaction begins by addition of the sulfur atom onto the acetylene triple bond which is susceptible to nucleophilic attack. Subsequently the intermediate (A) undergoes intramolecular cyclization to afford deliberated thiazolidin-4-one.

All the synthesized thiazolidin-4-ones have been characterized on the basis of elemental and spectral studies. 


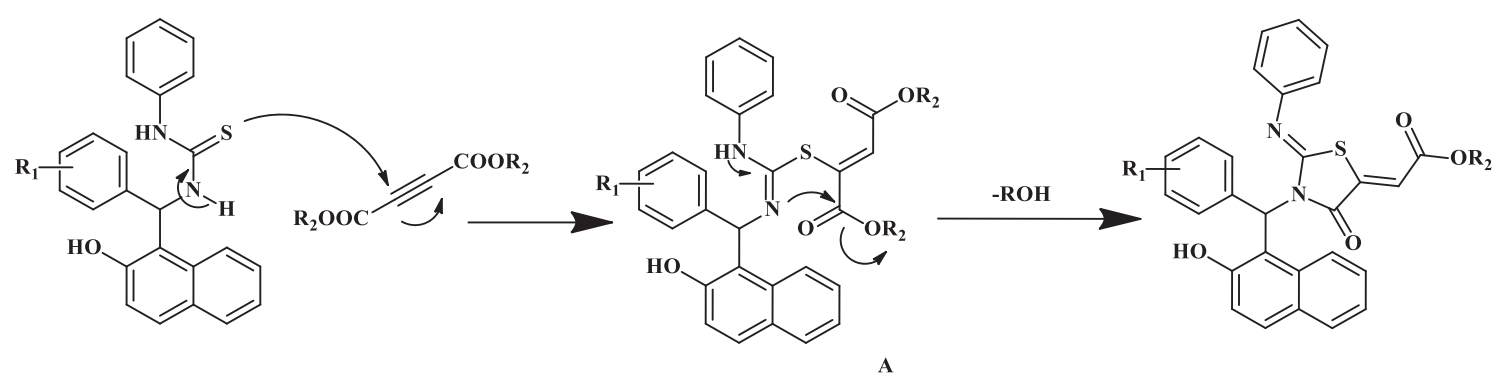

Scheme 3. A plausible mechanism for the formation of thiazolidin-4- ones.

The FT-IR spectrum of $\mathbf{2 a}$ revealed a broad band at $3430 \mathrm{~cm}^{-1}$ due to the absorption of $\mathrm{OH}$ group. Two strong absorption bands were also revealed at 1731 and $1684 \mathrm{~cm}^{-1}$ due to carbonyl groups. The ${ }^{1} \mathrm{H}$ NMR spectrum of 2a showed singlets at 3.94 and $6.58 \mathrm{ppm}$, which are due to the methyl and vinyl protons. The singlet at $7.09 \mathrm{ppm}$ was assigned to aliphatic hydrogen of aminoalkyl naphthol moiety $(\mathrm{CH})$ and the signal observed at $9.81 \mathrm{ppm}$ corresponds to the $\mathrm{OH}$ proton. Also, the ${ }^{1} \mathrm{H}$ NMR of $\mathbf{2 a}$ revealed signals between 7.08 and 7.95 with the integration of 16 protons which was consistent with protons of naphthyl ring and two phenyl rings. The ${ }^{13} \mathrm{C}$ NMR of this compound showed two characteristic peaks at 164.0 and $166.4 \mathrm{ppm}$ for carbonyl groups. The peaks at 52.9 and $66.1 \mathrm{ppm}$ correspond to the methyl and methine carbons of compound. The other signals in the range of $115.8-154.7 \mathrm{ppm}$ belong to the other $\mathrm{sp}^{2}$ carbons in the molecule.

\section{Conclusion}

In conclusion, an efficient, facile and environmentally benign synthesis of novel functionalized thiazolidin-4-ones has been achieved by the reaction of 1-thiocarbamidoalkyl-2-naphthols with dialkyl acetylenedicarboxylates in ethanol. The attractive features of this procedure are the mild reaction conditions, short reaction times, absence of any catalyst, high yields and simple isolation of products.

\section{Supplementary Information}

Supplementary Information associated with this article is available at www.ias.ac.in/chemsci.

\section{Acknowledgements}

We gratefully acknowledge the financial support from the Research Council of Shahid Bahonar University of Kerman.

\section{References}

1. Montgomery J 2000 Acc. Chem. Res. 33467

2. Shaterian H R, Yarahmadi H and Ghashang M 2008 Tetrahedron 641263

3. Soleimani E and Zainali M 2012 Synth. Commun. 42 1885

4. Wang M, Song Z G and Liang Y 2012 Synth. Commun. 42582

5. Zare A, Yousofi T and Moosavi Zare A R 2012 RSC Adv. 27988

6. Zare A, Akbarzadeh S, Foroozani E, Kaveh H, MoosaviZare A R and Hasaninejad A R 2012 J. Sulfur. Chem. 33 259

7. Eshghi H, Zohuri G H and Damavandi S 2012 Synth. Commun. 42516

8. Zhang Z P, Wen J M, Li J H and Hu W X 2009 J. Chem. Res. 3162

9. Srihari G, Nagaraju M and Murthy M M 2007 Helv. Chim. Acta 901497

10. Khan S A and Yusuf M 2009 Eur. J. Med. Chem. 442597

11. Ucho F D T, Cattani V B, Lima M C A, Galdino S L, Pitta I R and Costa T D 2008 J. Braz. Chem. Soc. 19 1553

12. Ottana R, Maccari R, Barreca M L, Bruno G, Rotondo A, Rossi A, Chiricosta G, DiPaola R, Sautebin L, Cuzzocread S and Gabriella Vigorita M 2005 Bioorg. Med. Chem. 134243

13. Zhou H, Wu S, Zhai S, Liu A, Sun Y and Li R 2008 J. Med. Chem. 511242

14. Ottanà R, Carotti S, Maccari R, Landini I, Chiricosta G and Caciagli B 2005 Bioorg. Med. Chem. Lett. 153930

15. Edwards P J 2008 Drug Discov. Today 131107

16. Vittoria Diurno M, Mazzoni O, Piscopo E, Calignano A, Giordano F and Bolognese A 1992 J. Med. Chem. 35 2910

17. Omar K, Geronikaki A, Zoumpoulakis P, Camoutsis C, Sokovic M and Iric A C 2010 Bioorg. Med. Chem. 18 426

18. Rawal R K, Tripathi R, Katti S B, Pannecouque C and De Clercq E 2007 Bioorg. Med. Chem. 151725

19. Bhandari S V, Bothara K G, Patil A A, Chitre T S, Sarkate A P, Gore S T, Dangre S C and Khachane C V 2009 Bioorg. Med. Chem. 17390

20. Ahmadi A, Saidi K and Khabazzadeh H 2009 Mol. Divers. 13353

21. Darehkordi A, Saidi K and Islami M R 2007 ARKIVOC 1180

22. Masoudi M, Darehkordi A and Moshrefi A 2010 World Appl. Sci. J. 101313 